Research Article

\title{
Application of Fuzzy Comprehensive Evaluation Based on Genetic Algorithm in Psychological Measurement
}

\author{
Lijun Chang \\ School of Management, Beijing Normal University, Zhuhai, Guangdong 519087, China \\ Correspondence should be addressed to Lijun Chang; changlijun2014@bnuz.edu.cn
}

Received 29 July 2021; Revised 17 September 2021; Accepted 23 September 2021; Published 25 October 2021

Academic Editor: Mian Ahmad Jan

Copyright (c 2021 Lijun Chang. This is an open access article distributed under the Creative Commons Attribution License, which permits unrestricted use, distribution, and reproduction in any medium, provided the original work is properly cited.

\begin{abstract}
Fuzzy comprehensive evaluation (FCE) is an artificial evaluation technique based on fuzzy mathematics, with real and accurate evaluation results that are extensively utilized in psychology and other fields. Hence, a technique is needed for assessing Likert scale data using fuzzy mathematics. This study examines the operators and weight distribution of the variables while individuals perform a complete and comprehensive evaluation and apply a genetic algorithm (GA) to determine the corresponding weight. Genetic processes based on population adaptation and evolution fundamentals have been hugely successful in solving problems and producing optimal solutions a long time ago. Furthermore, we measure psychological data from the Likert scale and analyze it using a fluid-based GA for a complete psychological assessment. Students of various genders used different compound operators and weight distribution to evaluate the instant noodle completely. This reflects the fact that the employment of a GA-based fuzzy comprehensive evaluation in psychological measurement and consumer psychology study is beneficial. The results show that the fuzzy comprehensive evaluation method based on the GA can effectively analyze the psychological measurement data of the Likert scale.
\end{abstract}

\section{Introduction}

The Likert scale is one of the most commonly used measurement tools in psychology [1]. When people analyze the data from the scale, they usually use the method of mathematical statistics. In other words, they think that the uncertainty of these data comes from randomness. However, there are many differences between psychological measurement and physical measurement. Because psychological measuring data is derived from the respondents' subjective reactions, particularly data acquired using the Likert scale, its uncertainty is higher [2]. Fuzziness and randomness are not the same. The uncertainty caused by randomness is due to the insufficient grasp of the causal relationship of things; that is, the conditions of things cannot be strictly controlled so that some accidental factors make the experimental results uncertain, but things themselves have obvious meanings [3]. The subjects' answers to intelligence test questions, for example, are frequently influenced by random factors and reflect a degree of uncertainty. It is appropriate to use the method of mathematical statistics to deal with these data. Fuzziness means that the limit of some things or concepts is not clear, which reflects the transition state between different concepts [4]. For example, the concepts of "comparative agreement" and "very agreement" in the Likert scale are fuzzy. Unfortunately, this fuzziness has not been properly studied in the current psychological measurement. People usually record these fuzzy concepts of the Likert scale as 1 to 5 points directly and then use the method of mathematical statistics to deal with it. This is not appropriate, because the uncertainty of the data obtained by the Likert scale is mainly reflected in the fuzziness, so whether the method of fuzzy mathematics can analyze this kind of psychological measurement data is a problem that needs to be discussed. The Likert scale is often used to score multiple variables in some areas of psychological measurement. Variables such as morality, ability, diligence, and performance, for example, are scored in personnel evaluation; in consumer psychology, the appearance, function, price, after-sales service, and other variables of the goods are scored. Multiple regression 
equations are often used in psychological statistics to describe the relationship between Likert scale scores obtained from multiple variables.

In fuzzy mathematics, the fuzzy relation equation reflects the results of fuzzy comprehensive evaluation [5]. In fuzzy comprehensive evaluation, the scores of multiple independent variables are transformed by some operator to get the scores of dependent variables, which is very similar to multiple regression equations. However, in the framework of fuzzy mathematics, the scores of independent variables are usually called single-factor evaluation, and the scores of dependent variables are called comment set scores. In fuzzy comprehensive evaluation, the main problem is the operator used, and there are four different operators [6]. People use the "maximum and minimum" operator or the "main factor determining" operator in the comprehensive evaluation, according to fuzzy mathematicians [7-10], but the hypothesis has yet to be validated in psychology. In the fuzzy comprehensive evaluation, it is also important to examine the kind of operator used.

A fuzzy comprehensive evaluation is a process of integrating multiple independent variable scores into a dependent variable score. The traditional method is to determine the weight by expert scoring, which is subjective. We use other objective methods to obtain the weight distribution. In the use of multiple regression equations, the least square method is usually used to determine the coefficients of each independent variable. These coefficients reflect the weight of each independent variable. It is an optimization problem to find the most suitable weight for the comprehensive evaluation of the subjects. A genetic algorithm (GA) simulates the evolution process of organisms and is a new global optimization method. It is used to figure out how much a fuzzy comprehensive examination is worth.

Not only should research be academically valuable, but it should also have a practical application. With the deepening impact of the current financial crisis on the real economy, the government has put forward some policies to expand domestic demand. If enterprises do not understand the needs of consumers, this is equivalent to selling products with their eyes closed. Therefore, it is very important to understand consumers' purchasing psychology and the weight of the impact of various attributes of goods on consumers.

According to the principle of market segmentation, enterprises should understand the different preferences of different target consumer groups for commodity attributes [11]. To find out whether they use the same operator in the fuzzy comprehensive evaluation, it is necessary to analyze the operator and weight distribution used by different subjects in the fuzzy comprehensive evaluation of goods. Based on the theoretical problems and practical requirements of psychological measurement described above, this study attempts to combine the genetic algorithm with a fuzzy comprehensive evaluation method by using the data from college students' evaluations of Master Kang's braised beef noodles as an example. Analysis of Likert data and customer preferences for various product features and operators was used in a complete fumigated assessment. The following are the main contributions of the proposed study:

(i) Firstly, we investigated related work in the field of psychological measurement and concluded that the best performance is achieved during psychological measurement.

(ii) Secondly, we chose the most accurate classification method, such as the genetic algorithm, as well as frequently used psychological assessment instruments, such as the Likert scale.

(iii) Thirdly, Likert scale variables have been widely used to determine actual data, from a huge quantity of college data of male and female students to weight distribution of each independent variable of psychological measurement via fuzzy evaluation.

(iv) Finally, the fuzzy relation equation reflects the results of a fuzzy comprehensive evaluation using Likert scale tools and a genetic algorithm to predict psychological measurement using fuzzy mathematics.

The remainder of the paper is organized as follows: Section 2 presents related research work; Section 3 depicts fuzzy comprehensive evaluation and genetic algorithm; Section 4 depicts empirical research, research objective, collected source code, research results, and experimental examination; and Section 5 discusses the operator in the fuzzy comprehensive evaluation method and application of the genetic algorithm. In the final section (i.e., Section 6), we conclude our paper.

\section{Related Work}

Psychological measurements, often known as psychological tests, are standardized assessments of a psychological characteristic like personality, intellect, or emotional functioning. They frequently comprise a sequence of true or false questions that respondents must answer. One of the most often used devices for assessing psychological exams is the Likert scale. Likert [12] proposed the scale, which comprises a set of questions that serve as markers of psychological measures. The scores in question, according to the author of [13], are built on an interval scale since they are obtained by psychological scaling. The psychological factors are assessed by the combined values of all interval questions [14]. Nevertheless, many scientists claimed that, in Likert, only facts structured in an ordinary scale are of course the option or the response. Regarding Likert, the interval variety of different levels has been claimed [15] to be not equal. The Likert scale thus is organized in an ordinal way. Analyzing data with added, subtracted, divided, or replicated data is improper. In addition, the analysis utilizing the arithmetical mean and the standard deviation [16] of such data is incorrect. As a result, assessing psychological variables by combining all items on the Likert scale is not appropriate. Furthermore, the authors in [17] noted that, in general, researchers would aggregate the scores from each item and 
then use the total scores to assess the variables, which is wrong because each item's weight is uneven.

Due to the difficulties described above, several attempts have been made to address this issue and develop an acceptable scale. Fuzzy logic is one of the approaches. It was created by [18] from a hazy set. The authors of [19] improved the Likert scale using fuzzy logic, resulting in a novel scale recognized as the fuzzy Likert scale. In this regard, the author in [20] linked the effectiveness of this scale to the Likert scale and discovered that measuring the variables using the fuzzy Likert scale was more precise than evaluating them using the conventional Likert scale.

By integrating the analytic hierarchical process (AHP) with the fuzzy evaluation technique, the authors of [21] presented a universal approach to usability evaluation. This technique develops a hierarchical index based on three major accessible qualities, product performance, efficiency, and customer satisfaction, by combining numerous sources of ambiguous information throughout product usability assessment.

Planning technology combines many fuzzy information sources to analyze in the process of product usability evaluation. The technology includes indicators such as accessible quality, product performance, efficiency, and customer satisfaction.

\section{Fuzzy Comprehensive Evaluation and Genetic Algorithm}

In this section, we explain fuzzy comprehensive evaluation and generic algorithms in detail.

3.1. Fuzzy Comprehensive Evaluation. The fuzzy comprehensive evaluation technique [15] is a mathematical approach for thoroughly evaluating things that are difficult to specify in the actual world utilizing fuzzy mathematics thinking and methodologies. Fuzzy mathematics has seen fast theoretical progress and widespread application for more than 30 years. The fuzzy comprehensive evaluation technique employs fuzzy mathematics' fuzzy set theory to completely evaluate systems. Information on the priority of various alternatives may be obtained as a guide for decisionmakers to make decisions using fuzzy evaluation. We need to first construct a fuzzy comprehensive evaluation index system while using the fuzzy comprehensive evaluation approach. The following essential ideas should guide the development of a fuzzy comprehensive assessment index system:

(i) There should be a comprehensive assessment index system

(ii) The evaluation index must be quantifiable and comparable in the evaluation index system

(iii) It is important to highlight the human aspect in the evaluation index and to completely integrate human variables for assessment in education

(iv) The assessment level in the assessment index should not be too thinly split
3.1.1. Fuzzy Set. There are many vague concepts in the objective world, such as "comparative agreement" and "very agreement" in the Likert scale. To express the fuzzy concept mathematically, American cybernetics expert Professor Zadeh put forward the concept of fuzzy set in 1965 and created a new discipline of fuzzy mathematics. The relationship between element $x$ and fuzzy set A breaches the membership degree limitation in fuzzy mathematics. In general set theory, this can only take 0 and 1 , but it may be extended to any value in the interval [0.1]. The membership function of $\mathrm{A}$ expresses the membership of element $x$ in the fuzzy set A.

The identification of the membership of a given problem is a major step towards resolving practical difficulties utilizing fuzzy mathematics. Five-point, three-point, fuzzy distribution, and fuzzy statistics approaches are among the ones proposed by experts both at home and abroad. In this study, a fuzzy statistical method will be used to get the membership function.

3.1.2. Elements of a Fuzzy Comprehensive Evaluation. A fuzzy comprehensive evaluation is to take fuzzy mathematics as a tool to make a comprehensive evaluation of something under the condition of considering a variety of factors. The evaluation model includes three elements [22]:

(1) Factor set $U=\left\{u_{1}, u_{2}, \ldots, u_{m}\right\}$

(2) Comments collection $V=\left\{v_{1}, v_{2}, \ldots, v_{n}\right\}$

(3) Single-factor judgment $f\left(u_{i}\right)=\left\{r_{i 1}, r_{i 2}, \ldots, r_{i n}\right\}, r_{i j}$ form fuzzy matrix $\mathrm{R}$ 。

In this study, the subjects made a comprehensive evaluation of Kang Shi Fu's braised beef noodles. Taste, appearance packaging, cake size, price, seasoning package, and advertising are among seven factors in the factor set. The comment set included five comments:

(i) Very dissatisfied

(ii) Relatively dissatisfied

(iii) Average

(iv) Relatively satisfied

(v) Very satisfied

Single-factor judgment is the evaluation of the seven factors mentioned above.

3.1.3. The Model of a Fuzzy Comprehensive Evaluation. The goal of the fuzzy evaluation model is to create a fuzzy mapping between each assessment component, such as effectiveness, competence, and user happiness, and a set of definite assessment scores, such as good or outstanding. The aim is to construct fuzzy sets for the assessment elements; for example, a given usability score, say 5 on a 7-point scale, may be assigned to both the outstanding scores. However, based on the weights assigned to each assessment component and the average scores given by various raters, the amount of each grade that the usefulness score corresponds to may differ. Figure 1 depicts the formal methods of our proposed fuzzy evaluation model. 


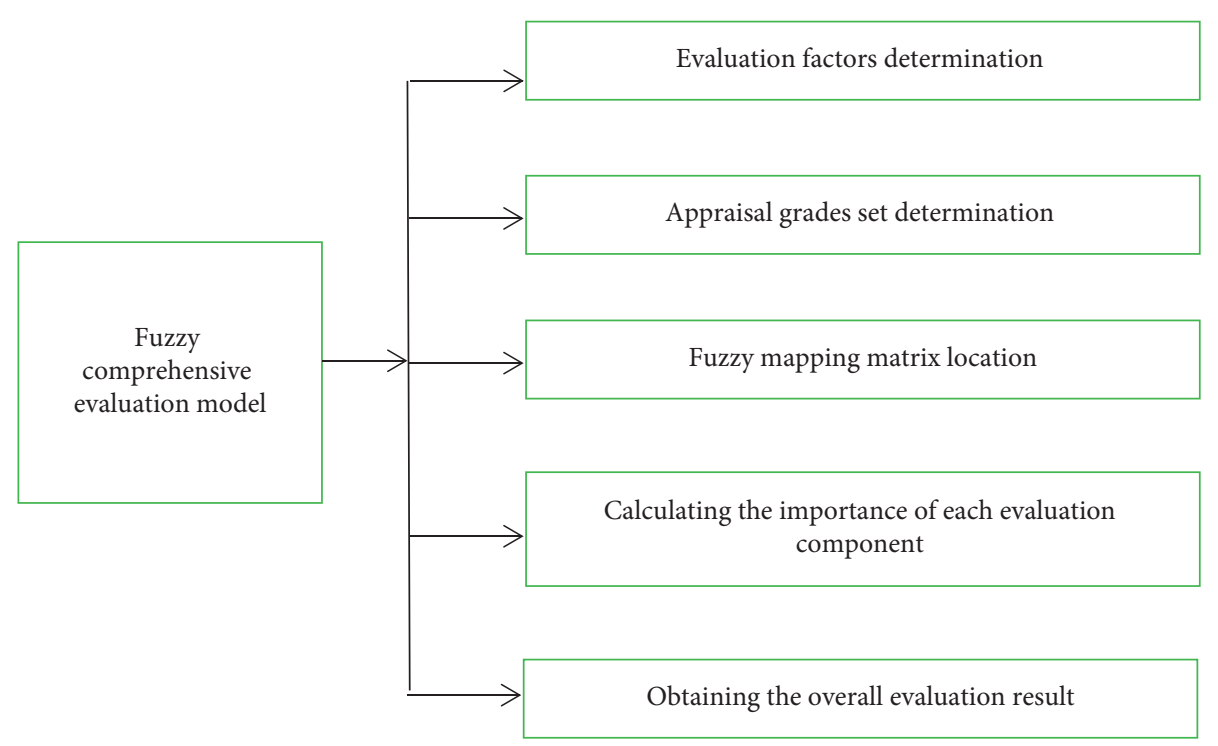

FIgURE 1: The fuzzy comprehensive assessment model's steps.

Step 1. Evaluation factors determination: the evaluation object, factor set, and comment set are all determined in the first phase. In this study, the evaluation object is Master Kang's braised beef noodles, and the factor set and remark set are described in the preceding section.

Step 2. Appraisal grades set determination: $V=v 1, v 2, \ldots, v m$, where $m$ is the number of levels in the appraisal, representing the appraisal set as a vector. For instance, if $m=5$, the assessment vector $\mathrm{V}=$ extremely poor, poor, medium, good, excellent might be expressed. In the second stage, expert consultation methods and analytic hierarchy procedures are used to create the weight distribution vector A of assessment factors. This weight distribution component is a tough topic, and experts frequently struggle to describe the weight in their brains with numbers; therefore, in this work, we attempt to estimate the weight vector using a genetic algorithm.

Step 3. Fuzzy mapping matrix location: the fuzzy comprehensive evaluation matrix $R$ is obtained in the third phase by evaluating each component.

Step 4. Calculating the importance of each evaluation component: the relative relevance of each assessment element in the overall product rating should be measured to get a thorough usability assessment. W, which can be constructed via the AHP technique, can represent the weight vector. As previously mentioned, weight may be expressed for $n$ evaluation factors by the vector $W=(W 1, W 2, \ldots, W n)$, which is equal to the sum of every element 1 . A composite method such as this is utilized during the fourth stage to obtain the comprehensive evaluation results $B$.

This is called the fuzzy relation equation, and it was developed by mathematicians who proposed four different operators, resulting in the four models that will be described later. The full assessment findings of Kang Shi Fu's braised beef noodles were collected directly using the Likert scale in this study. The task at hand is to solve the value of a from the fuzzy relation equation i.e., invert the fuzzy comprehensive evaluation, to obtain the weight vector.

Step 5. Obtaining the overall evaluation result: The total assessment outcome may be determined by considering the comparative weights of every evaluation component so that a single vector representing a similar level of assessment scores $m$ can be expressed as

$$
B=(b 1, b 2, b 3 \ldots b m)=W * R,
$$

where “*” is an arrangement operator.

The various processes in the composition impact how different weight distributions, i.e., in vector $W$, modify the final evaluation vector $B$. There is obvious overriding importance in the choice of the composition operators. We presume, for the current purpose, that all assessment variables have to be taken into account, such that there is no one aspect more picked or disregarded than others. We thus opt to utilize the operator composition which determines each member $B j$ of the last evaluation vector using the following formula which is suited for assessments that must accommodate every weight of the factors:

$$
B j=\min \left\{1, \sum_{i-1}^{n} \text { Wirij }\right\}(\text { where } I, \quad j=1,2,3 \ldots, n) .
$$

Rendering to the different composition operators, the following four models are obtained [6-10]:

(i) The main factor is decisive, and its operator is

$$
\begin{gathered}
M(\Lambda, V), \\
b_{k}=\Lambda_{k=1}^{m}\left(a_{k} \Lambda r_{k j}\right)=\max \left\{\min a_{k}, r_{k j}\right\} .
\end{gathered}
$$


The operator only considers the most important factor; other factors do not really work.

(ii) The main factor is type I, whose operator is

$$
\begin{aligned}
& M(., V), \\
b_{k}= & \Lambda_{k=1}^{m}\left(a_{k} \Lambda r_{k j}\right)=\max \left\{\min a_{k}, r_{k j}\right\} .
\end{aligned}
$$

The model is a little more accurate than the main factor determinant because it uses ordinary multiplication and considers all factors.

(iii) The main factor highlights type II, whose operator is $M(\wedge, \oplus)$, where $\oplus$ is a bounded sum, that is

$$
S_{1}^{\oplus}, S_{1}^{\oplus}, \ldots, S_{1}^{\oplus}=\min \left(1, \sum_{k=1}^{t} S_{k}\right) .
$$

Since the sum of the weights is equal to 1 ,

$$
b_{j}=\oplus_{k-1}^{m}\left(a_{k} \Lambda r_{k j}\right)=\sum_{k=1}^{m}\left(a_{k} \Lambda r_{k j}\right) .
$$

The model uses the method of summation, and the result is more accurate than that of the main factor determinant.

(iv) Weighted average type, whose operator is

$$
M(.,+), b_{j}=\sum_{k=1}^{m}\left(a_{k} \Lambda r_{k j}\right) .
$$

Theoretically, this model is more accurate than the above three models.

Even though mathematicians have proposed operators for these four types of composition operations, they have neglected to describe the types of operators and models that people employ in the comprehensive evaluation. There has not been an empirical study of psychology to assess it yet; therefore it is an issue that has to be addressed.

3.2. Genetic Algorithm. Although mathematicians proposed operators for these four combinatorial operations, they ignored the types of operators and models used by humans in their thorough review. There has not been any empirical psychological research to assess this, therefore it is a problem that has to be addressed. The genetic algorithm imitates the concept of biological evolution by applying genetic operators such as selection, crossover, and mutation to a population to generate a new generation of the population while seeking the best answer.

3.2.1. Elements of Genetic Algorithm. Imitating the diversity of biological evolution, people put forward a variety of coding methods and genetic operators of genetic algorithms. Goldberg summarized them as a unified genetic algorithm, called simple genetic algorithm (SGA) [23]. (i) Chromosome coding method

(ii) Individual fitness evaluation

(iii) Genetic operators, including selection, crossover, and mutation

(iv) Operation parameters including population size $m$, terminal evolution algebra $T$, crossover probability $\mathrm{P}_{-} \mathrm{c} \circ$, and variation probability $\mathrm{p}_{-} \mathrm{m}$ 。

The basic genetic algorithm can be defined as an 8-tuple as in (12).

$$
S G A=\left(C, E, P_{0}, M, \Phi, \Gamma, \Psi, T\right),
$$

where $C$ is the individual coding mode, $E$ is the individual fitness evaluation function, $P_{0}$ is the initial population, $M$ is the population size, selection operator, $\Gamma$ is the crossover operator, $\Psi$ is the mutation operator, and $T$ is the termination condition.

\subsubsection{Implementation of Genetic Algorithm}

(1) Coding Method. The method of transforming the feasible solution of a problem from its solution space to the search space that a genetic algorithm can handle is called coding, which is the primary problem when using the genetic algorithm to solve practical problems. The commonly coding methods include binary coding method, gray coding method, floatingpoint coding method, and symbol coding method. The binary coding method uses the symbol string composed of 0 and 1 to represent the individual. Gray code is a coding method in which only one code bit is different and the other code bits are identical between the coding values of two consecutive integers. In the floating-point coding method, each gene value of an individual is represented by a floating-point number in a certain range. That is, the true value of the variable is used to encode the data. Symbol coding means that gene values are taken from a symbol set with no numerical meaning but only code meaning.

People usually use different coding methods according to the nature of the problem to be solved. The floating-point code method is used in this study as it can represent a large range, provides the best precision solution, is efficient, and makes it easy to perform a genetic search in a large space. Let the optimization be the minimization problem by (13):

$$
\left\{\begin{array}{c}
\min f(x) \\
L(j) \leq x(j) \leq U(j)
\end{array}\right\}
$$

where $x=\{x(j)\}$ is the optimization variable set.

$[L(j), U(j)]$ is the change interval of $x(j)$, and $f$ in the objective function.

In floating-point coding, (14) is used as linear transformation.

$$
x(j)=L(j)+y(j)[U(j)-L(j)] .
$$

The $j^{\text {th }}$ variable $x(j)$ with initial change interval $[L(j), U(j)]$ is transformed into floating-point number $y(j)$ on $[0,1]$ interval, so that the value range of all variables is unified to $[0,1]$ interval. 
(2) Fitness Function. In a genetic algorithm, fitness is used to measure the degree to which an individual is helpful to find the optimal solution in optimization calculation. The function that measures individual fitness is called fitness function. The evolutionary process of a group is based on the fitness of each individual in the group. Through repeated iterations, the individual with the best fitness is constantly sought until the optimal solution or approximate optimal solution of the problem is obtained. To prevent premature phenomena and maintain the diversity of the population, it is necessary to expand or reduce the fitness of individuals in different stages of the genetic algorithm, which is called fitness scale transformation. The commonly used transformation methods are linear scale transformation, power scale transformation, exponential scale transformation, and ranking scale transformation. The ranking scale transformation is used in this study, which is based on everyone's fitness order rather than the score value. The most suitable individual number is 1 , followed by 2 and so on. The advantage of this transformation is that the scale value of the individual is proportional to the size of the population $n$, and the sum of the scale values of the whole population is equal to the number of parents of the next generation, which avoids the influence of the initial value limit [23].

(3) Selection Operator. In the process of biological heredity, the species with higher adaptability to the environment will have more chances to survive to the next generation. The selection operator is used in the genetic algorithm of survival of the fittest to carry out the operation of "survival of the fittest." The common selection operators are roulette, optimal saving strategy, deterministic sampling selection, and random selection without playback. In this study, we used the division ratio of the faceplate for segmentation. The probability of everyone being selected is directly proportional to their fitness.

(4) Crossover Operator. Crossover operator refers to the crossover operator used by two chromosome pairs in a certain way, including single-point crossover, two-point crossover, multipoint crossover, uniform crossover, and arithmetic crossover. In this study, we use a two-point crossover, that is, randomly set two crossover points in the coding string of two paired individuals and then exchange the chromosomes between the two crossover points.

(5) Mutation Operator. The mutation operator is used to replace the gene values of some loci in chromosome coding string with other alleles to form a new individual. The commonly used mutation operators are basic bit mutation, uniform mutation, boundary mutation, nonuniform mutation, and Gaussian mutation. In this study, Gaussian mutation is used to replace the original gene value with a random number under the standard normal distribution, which is more suitable for floating-point coding.

3.3. Fuzzy Comprehensive Evaluation Based on Genetic Algorithm. Compared with computers, people can often deal with fuzzy information better. One of the original purposes of establishing fuzzy mathematics is to use mathematical methods to describe people's fuzzy information processing process. A fuzzy comprehensive evaluation describes how people arrived at an overall evaluation of something by evaluating individual factors. Because it is difficult for people to accurately articulate the weight distribution in their heads, knowing how much each element costs is one of the keys. As a result, evaluating the weight value of each factor in a fuzzy comprehensive evaluation is very difficult. When the researchers cannot let the subjects express the weight distribution in their minds by the oral report method commonly used in psychology, they get the weight from other ways. The excellent optimization function of the genetic algorithm provides a good inspiration. The process of searching for weights is to search a group of values so that the results obtained by substituting them into the fuzzy relation equation are the most consistent with the actual response of the subjects. This is a process of searching for the optimal solution. Therefore, this study uses a genetic algorithm to search for a group of values that can represent the weight distribution in the brain of the subjects and uses the actual response values of the subjects for testing; the results are more reliable than those of the oral report method.

\section{Experimental Work and Simulation Results}

4.1. Experimental Work. In this portion of the study, we provide the experiments that were carried out as well as the simulation findings obtained during these studies. Many simulation experiments on the suggested psychological measurement were conducted utilizing fuzzy comprehensive evaluation based on the evolutionary algorithm. During our experimental work, we acquired the weight and fitness of male and female students' evaluations of various criteria under different operator models using the genetic algorithm, and we classified the weights of males and females into two categories: normalized weights and ranking weights. Table 1 shows the hardware requirements for the experimental work.

4.1.1. Parameter Setting. The following parameters are set in the GUI of the genetic algorithm, toolbox GADS:

(i) Population type: double-precision vector.

(ii) Population size: 100 .

(iii) Create function: uniform distribution.

(iv) Fitness scale transformation: ranking.

(v) Selection operator: roulette.

(vi) Number of elites: 4 .

(vii) Crossover rate: 0.80 .

(viii) Mutation operator: Gaussian mutation.

(ix) Crossing mode: two-point crossing.

(x) Migration: bidirectional migration.

(xi) Mixed function: fmincon. 
TABLE 1: Hardware requirements.

\begin{tabular}{lcc}
\hline S. no. & Hardware & Specification \\
\hline 1. & Computer & Dell laptop \\
2. & Operating system & Windows 10 \\
3. & Core & i7 \\
4. & Processor & $2.7 \mathrm{GHz}$ \\
5. & RAM & $32 \mathrm{~GB}$ \\
6. & Generation & $11^{\text {th }}$ \\
\hline
\end{tabular}

(xii) Constraint condition: lower bound: 0; upper bound: 1 .

(xiii) Termination condition: algebra: 1000 .

(xiv) Fitness limit: 0 .

(xv) Stagnation algebra: 100.
Other parameters are set to default values [23-27].

4.1.2. Experiment Steps. Factor set $U$ and comment set $V$ are as follows:

$U(u 1, u 2, u 3, u 4, u 5, u 6, u 7)=\{$ flavor, taste, appearance, packaging, cake size, price, seasoning package, advertising $\}$,

$V(v 1, v 2, v 3, v 4, v 5, v 6, v 7)=\{$ very dissatisfied, quite dissatisfied, average, quite satisfied, very satisfied.

Evaluation matrix of male students is as follows:

$$
R 1=\left[\begin{array}{ccccc}
0.36 & 0.50 & 0.12 & 0.02 & 0.00 \\
0.28 & 0.60 & 0.12 & 0.00 & 0.00 \\
0.20 & 0.48 & 0.20 & 0.12 & 0.00 \\
0.20 & 0.38 & 0.22 & 0.20 & 0.00 \\
0.12 & 0.40 & 0.30 & 0.16 & 0.02 \\
0.28 & 0.40 & 0.18 & 0.10 & 0.04 \\
0.32 & 0.40 & 0.16 & 0 . .08 & 0.04
\end{array}\right] .
$$

Evaluation matrix of female students is as follows:

$$
R 2=\left[\begin{array}{lllll}
0.46 & 0.39 & 0.12 & 0.01 & 0.03 \\
0.41 & 0.40 & 0.14 & 0.04 & 0.01 \\
0.21 & 0.39 & 0.37 & 0.04 & 0.00 \\
0.13 & 0.33 & 0.39 & 0.10 & 0.04 \\
0.13 & 0.25 & 0.39 & 0.17 & 0.05 \\
0.27 & 0.38 & 0.29 & 0 . .04 & 0.01 \\
0.27 & 0.37 & 0.35 & 0.00 & 0.00
\end{array}\right] .
$$

Comprehensive evaluation results of males are as follows:

$$
B 1=\left[\begin{array}{lllll}
0.23 & 0.34 & 0.20 & 0.18 & 0.05
\end{array}\right]
$$

Comprehensive evaluation results of female students are as follows:

$$
B 2=\left[\begin{array}{lllll}
0.25 & 0.42 & 0.23 & 0.05 & 0.05
\end{array}\right] .
$$

4.1.3. Fitness Function Program. According to the composition operator used in the four different models of the fuzzy comprehensive evaluation, the fitness function program was written by using MATLAB language. The Euclidean distance between the comprehensive evaluation results obtained by the genetic algorithm and the actual comprehensive evaluation results of the subjects is taken as the fitness $s$ as shown in the following equation:

$$
S=\sqrt{\sum_{j=1}^{m}(d(j)-q(j))^{2}}
$$

where $d(j)$ is the score of the $j^{\text {th }}$ grade obtained by the normalized genetic algorithm, $q(j)$ is the score of the $j^{\text {th }}$ grade obtained by the normalized subject's actual comprehensive evaluation, and $m$ is the number of evaluation grades.

\subsection{Results}

4.2.1. Operators of Composition Operation. The weight and fitness of male students' evaluation of various factors of Master Kang's braised beef noodles under different operator models obtained by the genetic algorithm are shown in Table 2 and in Figure 2.

It can be seen from the table that for males the fitness value of model 1 is the smallest, so it can be considered that males use the synthesis operator $m(a, V)$ of model 1 in a fuzzy comprehensive evaluation.

The weight and fitness of female students' evaluations of different factors of Master Kang's braised beef noodles using various operators' models developed using the genetic algorithm are shown in Table 3 and Figure 3. For female students, the fitness of model 3 is the smallest, so it can be 
TABLE 2: Weight and fitness under different operator models.

\begin{tabular}{lccccccrrrr}
\hline Gender & Model & W1 & W2 & W3 & W4 & W5 & W6 & W7 & Fitness \\
\hline \multirow{4}{*}{ Male } & 1 & 0.0525 & 0.2442 & 0.3707 & 0.2695 & 0.0991 & 0.2517 & 0.1446 & 0.0045 \\
& 2 & 0.0879 & 0.5328 & 0.5299 & 0.8577 & 0.6416 & 0.7983 & 0.1911 & 0.0173 \\
& 3 & 0.0057 & 0.0000 & 0.0181 & 0.5018 & 0.1600 & 0.0410 & 0.8855 & 0.0224 \\
& 4 & 0.0000 & 0.0000 & 0.0000 & 1.0000 & 0.0000 & 0.0364 & 0.2995 & 0.0616 \\
\hline
\end{tabular}

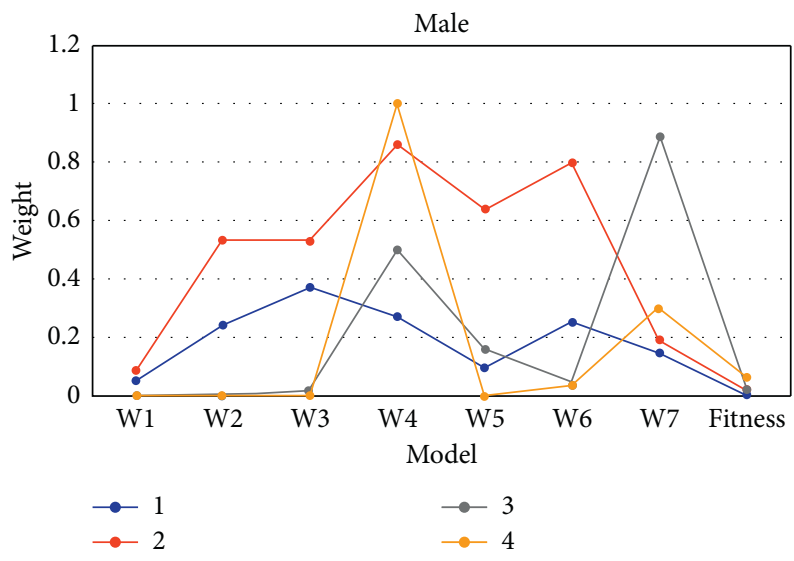

Figure 2: Comparison of weight and fitness for males, under different operator models.

TABLE 3: Weight and fitness under different operator models for females.

\begin{tabular}{ccccccccrr}
\hline Gender & Model & W1 & W2 & W3 & W4 & W5 & W6 & W7 & Fitness \\
\hline \multirow{4}{*}{ Female } & 1 & 0.1379 & 0.2337 & 0.260 & 0.0397 & 0.0484 & 0.5134 & 0.2761 & 0.0742 \\
& 2 & 0.3197 & 0.1034 & 0.3056 & 0.6168 & 0.0691 & 0.9992 & 0.5201 & 0.0787 \\
& 3 & 0.3864 & 0.7506 & 0.6823 & 0.3300 & 0.0329 & 0.0026 & 0.0000 & 0.0616 \\
& 4 & 0.0000 & 0.9957 & 0.2063 & 0.7032 & 0.0000 & 0.0003 & 0.0000 & 0.0721 \\
\hline
\end{tabular}

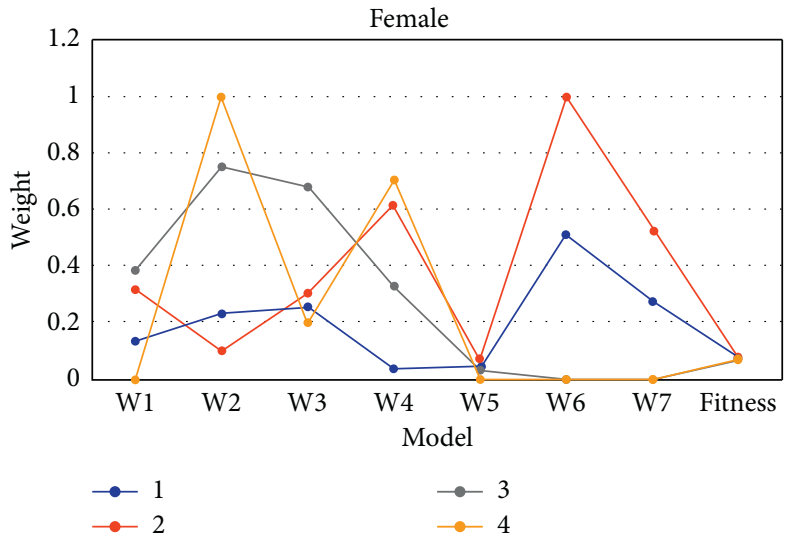

Figure 3: Comparison of weight and fitness for females, under different operator models.

considered that female students use the composition operator $m(a, R)$ of model 3 .

4.2.2. A Fuzzy Comprehensive Evaluation of the Weight. The weights of males in model 1 and females in model 3 in Table 1 and Table 2 are normalized, respectively, and the data are shown in Table 4 and Figure 4.
We arranged the data in Table 4 according to the weight and got the weight order of each factor, as shown in Table 5.

The normalized weight of males and females is shown in Figure 4. From W1 through W7, the normalized weights are displayed. This means that the males' weight during $\mathrm{W} 1$ is 0.0367, while the females' weight is 0.1769 . Similarly, the normalized weight W2 is 0.1705 for males and 0.3436 for females, and so on. 
TABLE 4: Normalized weight.

\begin{tabular}{lccccccc}
\hline Gender & W1 & W2 & W3 & W4 & W5 & W6 & W7 \\
\hline Male & 0.0367 & 0.1705 & 0.2588 & 0.1882 & 0.0692 & 0.1757 & 0.101 \\
Female & 0.1769 & 0.3436 & 0.3123 & 0.151 & 0.151 & 0.0012 & 0 \\
\hline
\end{tabular}

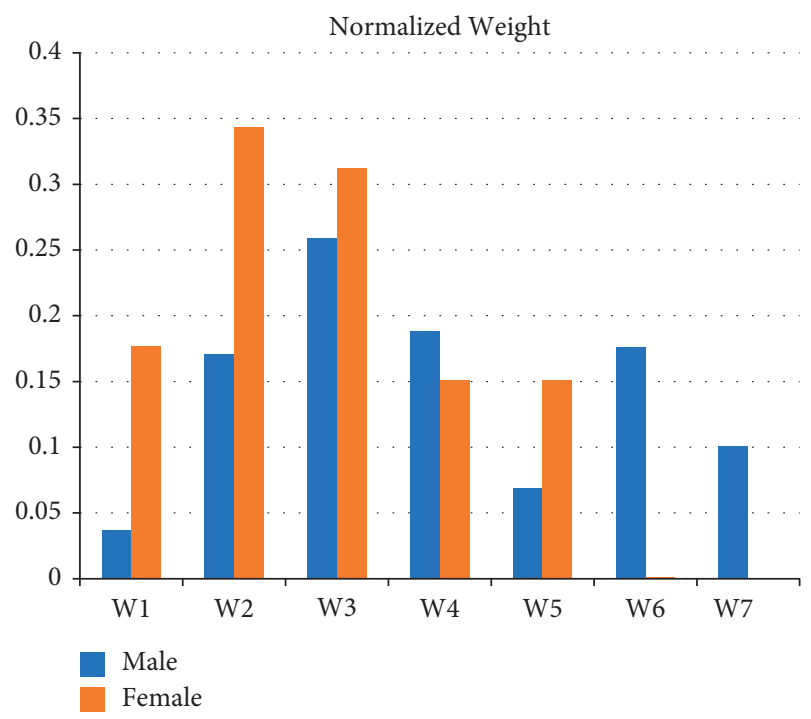

FIGURE 4: Normalized weight for males and females.

TABLE 5: Ranking of weights.

\begin{tabular}{lccccccc}
\hline Gender & W1 & W2 & W3 & W4 & W5 & W6 & W7 \\
\hline Male & 7 & 4 & 1 & 2 & 6 & 3 & 5 \\
Female & 3 & 1 & 2 & 4 & 5 & 6 & 7
\end{tabular}

Both males' and females' ranking weights are shown in Figure 5. From W1 through W7, the ranking weights are displayed. This means that for W1 the males' weight rank is 7 and the females' weight rank is 3, respectively. Similarly, for ranked weight W2, male weight is rated 4 and female weight is ranked 1 , and so on.

\section{Discussion}

5.1. Data Sources. Based on the discussion and questionnaire survey, the instant noodles questionnaire was compiled to investigate the evaluation of students from three universities, including Nanjing Normal University in Nanjing. The subjects were asked to evaluate five separate brands of instant noodles: Master Kong, Uni-President, Jinmailang, Fumanduo, and Rising. With a Likert 5-point scale, they scored their purchase intention from eight aspects: flavor, taste, price, seasoning bag, size of dough, appearance, packaging, and advertising. One-point evaluation means "very reluctant to buy," and five-point evaluation means "very willing to buy." Each brand includes some representative products; for example, Master Kang brand includes braised beef noodles, crab roe lion head, and scallion oil noodles; unified brand includes Haojindao braised beef flavor, Haojindao sauerkraut shredded meat flavor, and Qiaomianguan sour bean beef taste, with a total of 22 varieties of instant noodles. After the preliminary

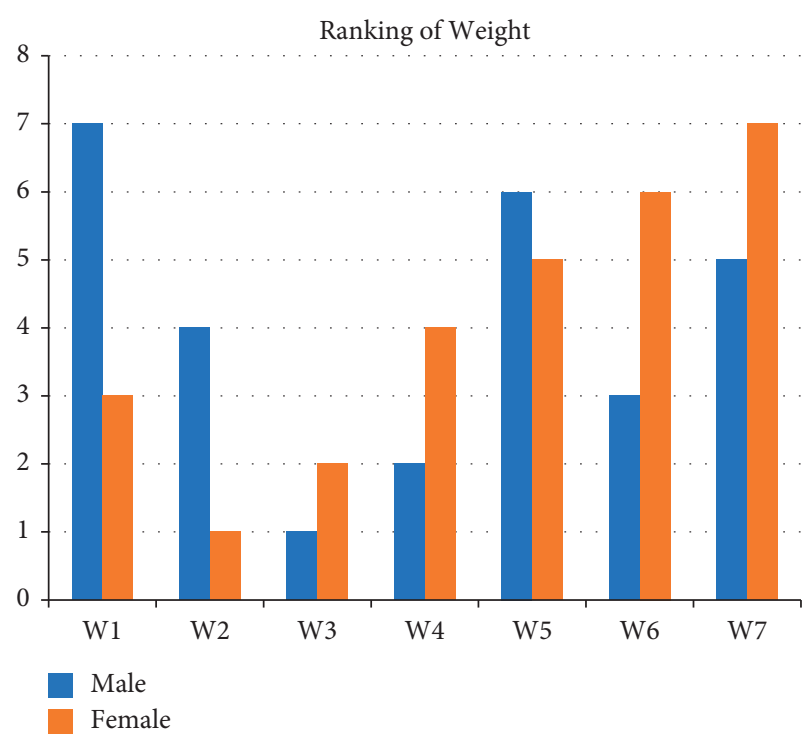

Figure 5: Male and female ranking of weights.

collation of the data, some questionnaires with incomplete data and careless answers were deleted, and 643 valid questionnaires were obtained, including 287 males and 356 females. As a preliminary study of fuzzy comprehensive evaluation based on genetic algorithm in psychological measurement, this paper only analyzes the evaluation data of these college students on Master Kang's braised beef noodles.

\subsection{The Use of a Fuzzy Comprehensive Evaluation Method to} Evaluate Likert Scale Data Processing. Due to the fuzziness of the subjects' answers from the Likert scale, this study uses the method of a fuzzy comprehensive evaluation to process these data. The Euclidean distance between the theoretical data and the actual data obtained by the fuzzy comprehensive evaluation model is 0.0045 for males, according to an example of college students' evaluation of Master Kang's braised beef noodles. The Euclidean distance between theoretical and actual data for females is 0.0045 , while the distance between the two groups of data is 0.0616 . That is, the difference between the theoretical and actual data is very small, showing that the fuzzy comprehensive evaluation method can be used to process Likert scale data.

\subsection{On the Composition Operator in the Fuzzy Comprehensive} Evaluation Method. The conclusion that the fuzzy comprehensive evaluation method can be applied to the data processing of the Likert scale is based on finding the appropriate synthesis operator. It can also be seen from the above calculation results that the Euclidean distance of female data is larger than that of male data, which indicates that a better operator can be found for female data. In essence, the operators of synthetic operations reflect the different characteristics of human cognitive activities in the process of information processing. Therefore, the joint efforts of psychometricians and cognitive psychologists are needed to find better operators. 
This study found that males and females use different operators. For the evaluation of Master Kang's braised beef noodles, males use the "max-min" operator in model 1, which only takes the maximum of $m$ as a constant, thus eliminating other factors. This indicates that those males have relatively simple thinking when deciding whether to buy, often only considering the most important factor; if the evaluation of this factor is high, it will produce purchase intention. Females use the operator in model 3, using "bounded sum" (i.e., "addition") instead of the "maximum" operation in model 1. That is to say, all factors have the opportunity to participate in the overall evaluation, and the role of the main factor is not as prominent as model 1. This also shows that those females should think more comprehensively than males when deciding whether to buy. This analysis result is quite consistent with the actual situation.

5.4. Application of the Genetic Algorithm. In this study, a genetic algorithm is used to invert the weight of fuzzy comprehensive evaluation. Genetic algorithm is an intelligent technology of global optimization, which has been successfully applied in the field of computer science and engineering technology, but it is rarely applied in psychological measurement. In the comprehensive evaluation, the weight reflects people's different emphasis on various factors. In essence, it is a psychological problem, but it is difficult for the subjects to express the weight distribution in their mind. This study used a genetic algorithm to search the weight distribution of the subjects' evaluation of Master Kang's braised beef noodles in the whole solution space. This provides a new method for similar research of psychology in the future. However, because the operation of a genetic algorithm is quite complex and many parameters need to be set, this study is to find the more appropriate parameters after many times of debugging. The genetic algorithm itself is still in continuous development, which requires psychologists to conduct more in-depth study and research when applying this method to psychological measurement.

5.5. On Weight Distribution. In this study, a genetic algorithm was used to get the weight distribution of fuzzy comprehensive evaluation of Master Kang's braised beef noodles by males and females. From the calculation results (Table 4), the first two factors males attach most importance to are "appearance package" and "size of dough cake," and the least important factor is "taste," followed by "price." This also reveals that males' thinking when buying instant noodles is relatively simple, if they look at the appearance package from the surface. Whether the "size of the cake" is satisfied or not will decide whether to buy or not, while the "taste," "price," and other substantive factors are largely ignored. It can also be seen from Table 4 that the first two factors that females attach most importance to are "taste" and "appearance packaging," and the last factor is "advertising," followed by "seasoning package." This also shows that those females are more careful and comprehensive than males in purchasing instant noodles. Although they also attach importance to "appearance packaging," they pay more attention to "taste." They believe in their own "taste," but do not believe in external advertising. In addition, it can be seen from Table 3 that the weight of "taste" and "taste" of female students is much higher than that of male students. Female students pay more attention to their subjective taste in the purchase of instant noodles, which also provides good data for psychologists to study the consumption psychology of different gender subjects.

\section{Conclusion}

The weight vector of the distribution must be determined before the classification issue could be solved with FCE. This research proposed a fuzzy comprehensive evaluation based on genetic algorithms. It outperforms several current Likert scale approaches when it comes to enhancing the efficiency of weight vector computations. The experiments proved that the fuzzy comprehensive evaluation method based on the genetic algorithm can be used to analyze the psychological measurement data of the Likert scale and get the composite operator used by the subjects and the weight distribution of each factor. Furthermore, different genders of college students use different synthetic operators in the fuzzy comprehensive evaluation of instant noodles, and their weight distribution is also different. Additionally, the fuzzy comprehensive evaluation method based on genetic algorithm is valuable for the research of psychological measurement and consumption psychology and meaningful for enterprises. [28].

\section{Data Availability}

The data used to support the findings of this study are available from the author upon request.

\section{Conflicts of Interest}

The author declares no conflicts of interest.

\section{Acknowledgments}

This study was supported by Beijing Normal University Teachers' Research Ability Promotion Project, Zhuhai, under Grant no. GL20180105.

\section{References}

[1] L. Crocker and J. Algina, Y. Jin, Introduction to Classical and Modern Test Theory, East China Normal University Press, Shanghai, China, 2004.

[2] Y. Li, "Psychological stress detection and early warning system based on wireless network transmission," Scientific Programming, vol. 20219 pages, Article ID 3739045, 2021.

[3] D. E. Goldberg, Genetic Algorithms in Search: Optimization and Machine Learning, Addison-Wesley Publishing, Boston, MA, USA, 1989.

[4] H. De Groote and S. C. Kimenju, "Comparing consumer preferences for color and nutritional quality in maize: application of a semi-double-bound logistic model on urban consumers in Kenya," Food Policy, vol. 33, no. 4, pp. 362-370, 2008. 
[5] H. Hwang, Y. Takane, and W. S. DeSarbo, "Fuzzy clusterwise growth curve models via generalized estimating equations: an application to the antisocial behavior of children," Multivariate Behavioral Research, vol. 42, no. 2, pp. 233-259, 2007.

[6] Y. J. Lei, S. W. Zhang, X. W. Li, and C. M. Zhou, MATLAB Genetic Algorithms Toolbox and its Applications, Xidian University Press, Xian, China, 2005.

[7] Z. Qiu-Qiang, L. Wen-Jun, F. Wan-Yi, and Z. Qi-Zhe, "College students' self-acceptance: a paint therapy group counseling intervention," Scientific Programming, vol. 2021, Article ID 5180607, 9 pages, 2021.

[8] B. S. Liang and D. L. Cao, Fuzzy Mathematics and its Applications, Science Press, Beijing, China, 2007.

[9] Z. Liang, K. Yang, Y. Sun, J. Yuan, H. Zhang, and Z. Zhang, Decision support for choice optimal power generation projects: fuzzy comprehensive evaluation model based on the electricity market," Energy Policy, vol. 34, no. 17, pp. 3359-3364, 2006.

[10] Z. M. Liu, M. E. Wu, and Y. Xu, "A method of weight conformation based on genetic algorithm," Journal of Wiayi University (Natural Science Edition), vol. 20, no. 3, pp. 45-48, 2006.

[11] S. K. Qin, Comprehensive Evaluation Theory and Applications, Publishing House of Electronics Industry, Beijing, China, 2003.

[12] R. A. Likert, "A technique for the measurement of attitudes," Archiv für Psychologie, vol. 40, p. 5e53, 1932.

[13] A. L. Edward, Techniques of Attitude Scale Construction, Prentice-Hall, Hoboken, NJ, USA, 1957.

[14] S. Tirakanan, Construction of an Instrument to Measure Variables in Social Sciences Research: Guidelines to Practice, Chulalongkorn University, Bangkok, Thailand, in Thai, 2008.

[15] L. Cohen, L. Manion, and K. Morrison, Research Methods in Education, Routledge Falmer, London, UK, 2000.

[16] C. Clegg, Simple Statistics, Cambridge University Press, Cambridge, England, 1998.

[17] K. Sukasem and S. Prasitratsin, A Handbook for Application of Lisrel Program, Samlada, Bangkok, Thailand, in Thai, 2007.

[18] L. A. Zadeh, "Fuzzy set," Information and Control, vol. 6, Article ID 338e353, 1965.

[19] M. Lalla, G. Facchinett, and G. Mastroleo, "Ordinal scale and fuzzy set systems to measure agreement: an application to the evaluation of teaching activity," Quality and Quantity, vol. 38, Article ID 577e601, 2004.

[20] Q. Li, "A novel Likert scale base on fuzzy set theory," Expert Systems with Applications, vol. 40, no. 5, Article ID 1609e1618, 2013.

[21] R. Zhou and A. H. S. Chan, "Using a fuzzy comprehensive evaluation method to determine product usability: a proposed theoretical framework," Work, vol. 56, no. 1, pp. 9-19, 2017.

[22] X. P. Wang and L. M. Cao, Genetic Algorithms: Theory Applications and Software, Xian Jiaotong University Press, Xian, China, 2002.

[23] K.-L. Wen, "A Matlab toolbox for grey clustering and fuzzy comprehensive evaluation," Advances in Engineering Software, vol. 39, no. 2, pp. 137-145, 2008.

[24] Y. C. Ye, L. H. Ke, and D. Y. Huang, Systematic Comprehensive Evaluation Technique and its Applications, Metallurgical Industry Press, Beijing, China, 2006.

[25] S.-C. Yu and M.-N. Yu, "Fuzzy partial credit scaling: a valid approach for scoring the beck depression inventory," Social Behavior and Personality: An International Journal, vol. 35, no. 9, pp. 1163-1172, 2007.
[26] M. Zhou and S. D. Sun, Genetic Algorithms: Theory and Applications, National Defense Industry Press, Beijing, China, 1999.

[27] C. Shao, "The implication of fuzzy comprehensive evaluation method in evaluating internal financial control of enterprise," International Business Research, vol. 2, no. 1, 2009.

[28] M. Fang, Y. Chen, R. Xue et al., "A hybrid machine learning approach for hypertension risk prediction," Neural Computing \& Applications, vol. 3, pp. 1-11, 2021. 